Accepted refereed manuscript of:

Bracci S, Bracci S, Cavina-Pratesi C, Connolly JD \& Ietswaart M (2016) Representational content of occipitotemporal and parietal tool areas, Neuropsychologia, 84, pp. 81-88.

DOI: 10.1016/j.neuropsychologia.2015.09.001

(c) 2016, Elsevier. Licensed under the Creative Commons AttributionNonCommercial-NoDerivatives 4.0 International

http://creativecommons.org/licenses/by-nc-nd/4.0/ 


\section{Author's Accepted Manuscript}

Representational content of occipitotemporal and parietal tool areas

Stefania Bracci, Cristiana Cavina-Pratesi, Jason D. Connolly, Magdalena Ietswaart

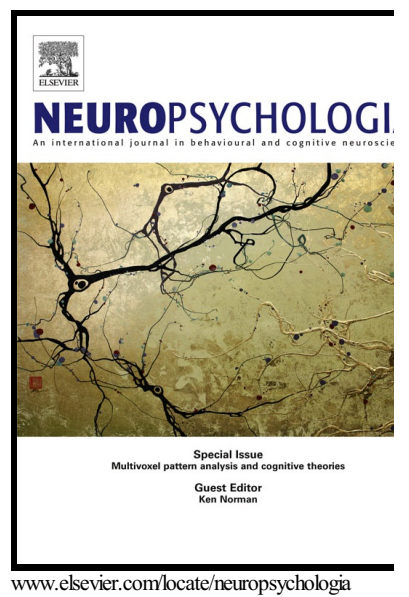

PII: S0028-3932(15)30145-7

DOI: $\quad$ http://dx.doi.org/10.1016/j.neuropsychologia.2015.09.001

Reference: NSY5713

To appear in: Neuropsychologia

Received date: 13 March 2015

Revised date: 31 August 2015

Accepted date: 1 September 2015

Cite this article as: Stefania Bracci, Cristiana Cavina-Pratesi, Jason D. Connoll: and Magdalena Ietswaart, Representational content of occipitotemporal an parietal tool areas, Neuropsychologia http://dx.doi.org/10.1016/j.neuropsychologia.2015.09.001

This is a PDF file of an unedited manuscript that has been accepted fo publication. As a service to our customers we are providing this early version o the manuscript. The manuscript will undergo copyediting, typesetting, an review of the resulting galley proof before it is published in its final citable form Please note that during the production process errors may be discovered whic could affect the content, and all legal disclaimers that apply to the journal pertain 


\title{
Representational content of occipitotemporal and parietal tool areas.
}

\author{
Stefania Bracci ${ }^{1}$, Cristiana Cavina-Pratesi ${ }^{2}$, Jason D. Connolly ${ }^{2}$, and Magdalena letswaart ${ }^{3}$, \\ ${ }^{1}$ Brain and Cognition, KU Leuven, 3000 Leuven, Belgium; \\ ${ }^{2}$ Department of Psychology, Durham University, Durham, United Kingdom; \\ ${ }^{3}$ Department of Psychology, Stirling University, Scotland, United Kingdom;
}

Running title:

The tool-network's representational content.

Corresponding author:

Stefania Bracci

phone num: +32 16377744

email: stefania.bracci@kuleuven.be

1) MVPA showed representational content differences in regions within the tool network.

2) Left parietal regions encode hand-tool action-related information.

3) Ventral OTC of the tool-network encodes object category information.

4) Left lateral OTC encodes hand-tool action-related and category-related information.

5) Left lateral OTC bridges ventral OTC (category) and parietal (action) representations. 


\section{Abstract}

It is now established that the perception of tools engages a left-lateralized network of frontoparietal and occipitotemporal cortical regions. Nevertheless, the precise computational role played by these areas is not yet well understood. To address this question, we used functional MRI to investigate the distribution of responses to pictures of tools and hands relative to other object categories in the so-called "tool" areas. Although hands and tools are visually not alike and belong to different object categories, these are both functionally linked when considering the common role of hands and tools in object manipulation. This distinction can provide insight into the differential functional role of areas within the "tool" network. Results demonstrated that images of hands and tools activate a common network of brain areas in the left intraparietal sulcus (IPS), left lateral occipitotemporal cortex (LOTC) and ventral occipitotemporal cortex (VOTC). Importantly, multivoxel pattern analysis revealed that the distribution of hand and tool response patterns in these regions differs. These observations provide support for the idea that the left IPS, left LOTC and VOTC might have distinct computational roles with regard to tool use. Specifically, these results suggest that while left IPS supports tool action-related computations and VOTC primarily encodes category specific aspects of objects, left LOTC bridges ventro occipitotemporal perception-related and parietal actionrelated representations by encoding both types of object information.

Key words: tools, action network, occipitotemporal cortex, parietal cortex, fMRI. 


\section{Introduction}

Tools can be conceptually defined by their specific action-related properties. These properties include the way that a particular tool is grasped and its particular translational profile during action deployment. Neuropsychological data would suggest that the different aspects of toolrelated information is processed in anatomically distinct brain regions. For example, lesions in occipitotemporal areas induce profound impairment(s) in object shape recognition and this impairment can even be selective to man-made objects (e.g., tools; see for review Capitani et al., 2003). Nevertheless, the ability to employ unrecognized objects correctly is spared in these patients (Sirigu et al., 1991). Yet a dissociation between a particular object's function and the object's actual manipulation has also been reported; temporal lesions induce loss of conceptual and functional knowledge for manipulable objects (Tranel et al., 2003; Damasio et al., 2004; Kalenine et al., 2010), whereas lesions in the inferior parietal lobule induce substantial impairment in the ability to manipulate objects according to their precise function (Sirigu et al., 1995; Buxbaum et al., 2000; Buxbaum et al., 2007). In accordance with the clinical literature, there is now a vast body of neuroimaging evidence that indicates the existence of a leftlateralized network of tool-selective regions. This network includes the lateral occipitotemporal (Chao et al., 1999), frontoparietal (Chao and Martin, 2000; Mahon, 2013) and ventral occipitotemporal (Chao et al., 1999; Mahon et al., 2007) areas. Although neural activation in these regions has been reported during both perceptual (Martin et al., 1996; Beauchamp et al., 2002, 2003; Beauchamp et al., 2004; Creem-Regehr and Lee, 2005; Creem-Regehr et al., 2005; Downing et al., 2006; Lewis, 2006; Almeida et al., 2013; Gallivan et al., 2013; Mahon et al., 2013; Peelen et al., 2013), and action-related tasks (Choi et al., 2001; Kellenbach et al., 2003; JohnsonFrey et al., 2005; Gallivan et al., 2013), the precise functional role of each of these regions in relation to tool-use is not yet well understood. 
It is tacitly understood that two brain areas with different fMRI patterns for two (or more) object categories should then provide for different computational roles. Conversely, a high degree of similarity in response patterns to two (or more) object categories provides support for parallel roles of these same brain areas. In order to disambiguate the computational roles of the different cortical areas within the well-established tool-selective brain regions, we investigated similarities (and differences) across response patterns to tools and hands and compared these with other object categories. The rationale is as follows: tools are inanimate yet action-related objects, whereas hands represent a self-animated body part largely involved in most body-related actions (e.g., object manipulation). Such a distinction can then assist to characterize the functional profile of the regions of the "tool" network. In other words, although hands and tools belong to distinct object domains (animate versus inanimate), these are functionally related within the action domain. We therefore predicted that there would be an overlapping distribution of hand and tool response patterns in brain areas involved in hand-tool action processing. Conversely, in those brain areas that encode object category information the response patterns to hands and tools should be highly distinct, as they are more closely related to the animate or inanimate domains respectively. Such insight would however refine the wellaccepted definition of the "tool" network, taking into account the differential computational content of each of the regions within the network. 


\section{Materials and methods}

Participants: Sixteen naive volunteers were functionally scanned (fMRI) in the present study and all participants provided informed consent prior to entering the scanning environment. The study was approved by the Ethical Committees of The School of Psychology and Sport Sciences of Northumbria University and Newcastle Magnetic Resonance Centre, School of Clinical Medical Sciences, University of Newcastle upon Tyne. All subjects were determined to be righthanded (Edinburgh Handedness Inventory; Oldfield, 1971). Consequent to excessive head motion being ascertained during post hoc analyses, one participant was excluded from further analyses.

Experimental design and stimuli: The present fMRI study consisted of two functional runs lasting 7 minutes 14 seconds and these corresponding to 217 functional volumes per run. Five distinct conditions were included in the present study: hands, tools, bodies, nonmanipulable objects and scrambled objects (Figure 1). Within each run the five stimulus categories were organized into five pseudo-random sequences of five stimulus blocks, and these were then each interleaved with fixation blocks each lasting $14 \mathrm{~s}$ in duration. The fixation blocks also appeared at the beginning and at the end of each run. Within each stimulus block, stimuli were centrally presented for 800 ms with a blank interstimulus interval (ISI) of $200 \mathrm{~ms}$. Each stimulus condition consisted of 70 greyscale images ( $400 \times 400$ pixels) on a white background. Stimulus presentation was controlled by a PC computer and the Psychophysics Toolbox software (Brainard, 1997) via Matlab (Mathworks, Natick, MA, USA). The pictures were projected (Canon Xeed SX6 projector) onto a screen located at the foot end of the scanner bed and the screen was viewed through a mirror mounted directly on the head coil. Stimuli were presented centrally and were each $12^{\circ} \times 12^{\circ}$ in visual angle. All participants performed a 1-back repetition detection 
task (either 1 or 2 repetitions were presented within a block) in order to ensure that attention was maintained throughout the duration of the fMRI scanning.

Imaging acquisition and preprocessing: All functional and structural images were acquired using a Phillips Achieva 3T scanner with an 8-channel head coil at the Newcastle Magnetic Resonance Centre, University of Newcastle-upon-Tyne (UK). Functional scans were gradient-echo echoplanar T2*-weighted images (EPI). Acquisition parameters were as follows: repetition time (TR) of 2s, echo time (TE) of 30ms, flip angle (FA) of 90 degree, field of view (FoV) of $192 \mathrm{~mm}$ and a matrix size of $64 \times 64$ pixels. Each volume consisted of 30 axial slices with $4.0 \mathrm{~mm}$ thickness and with no gap between slices. The structural scans had a TR of 9.6, a TE of 4.6, a FA of $8 \mathrm{deg}$, a FoV of $256 \mathrm{~mm}$ and a matrix of 256 x 208 pixels with 180 slices of $1.0 \mathrm{~mm}$ thickness. Data preprocessing and analyses were performed using Brain Voyager QX (version 2.20; Brain Innovation, Maastricht, The Netherlands) and MatLab (Mathworks, Natick, MA, USA). Preprocessing of the functional data included three-dimensional head-motion correction, linear trend removal, high-pass temporal filtering (cutoff 3 cycles per time course) and spatial smoothing (6-mm full-width half-maximum isotropic Gaussian kernel). For all participants, the functional images were subsequently co-registered to the T1 anatomical images. All of the anatomical images were subsequently transformed into Talairach stereotaxic space. This transformation was applied to the aligned functional data, which was subsequently resampled to $1 \mathrm{~mm}^{3}$ isotropic voxels.

Statistical analysis: Data were analyzed using a general linear model (GLM) random-effects group-averaged analysis. The GLM model was computed for each participant and this included the 5 conditions of interest and the 6 parameters to account for participant head motion. The 
fixation blocks represented the baseline condition. The GLM predictors' time courses were modeled using a linear model of the blood-oxygen-level dependent (BOLD) hemodynamic response using the default Brain Voyager QX "two-gamma” function. Prior to computing the GLM analysis, all functional runs were further z-normalized. Given the large body of evidence that suggests a left lateralized tool network (for review, see Lewis 2006), the primary data analyses were performed for areas in the left hemisphere. Furthermore, additional analyses were carried out for areas in the right hemisphere and these are reported in the

\section{Supplementary Figure 1-2 and Supplementary results.}

In the region-of-interest (ROI) analysis, the tool- and hand-responsive ROls were identified in each individual subject in the left hemisphere by comparing pictures of hands (or tools) versus pictures of scrambled objects. Our statistical activation maps were thresholded at $p<0.005$ (uncorrected) and these ROls were restricted to a $20 \mathrm{~mm}^{3}$ cube centered on the voxel with the highest signal peak. When no active voxels were found at this threshold, a more liberal threshold of $p<0.01$ was applied. For each condition and for each ROI, parameter estimates were extracted from the average of both runs (run 1 and run 2) and then further tested using analyses of variance (ANOVAs) and post-hoc pairwise t-tests. In LOTC, both the tool and the hand contrast could be localized in all participants. Conversely, in IPS and VOTC, both contrasts could not be defined in three participants. These participants were therefore excluded from the ROI analysis in each of these ROIs.

Correlation-based multivoxel pattern analysis (MVPA; Haxby et al., 2001) was carried out to investigate the similarity in the response patterns to hands, tools, bodies and nonmanipulable objects in all of the identified ROI-based occipitotemporal and parietal tool areas. For the MVPA analysis, ROIs were defined in the intraparietal sulcus (IPS), lateral occipitotemporal (LOTC) and ventro occipitotemporal (VOTC) cortex in the left hemisphere 
contrasting (at the group level) hands, tools, bodies and nonmanipulable objects relative to scrambled objects (uncorrected threshold, $p<0.001$ ). These ROls were demarcated by selecting all active voxels within a cube of $30 \mathrm{~mm}$ width centered on the voxel with the highest peak activation. For each participant, parameter estimates were extracted for each voxel and each condition, separately for run 1 (odd) and run 2 (even). Multivoxel activity patterns for each stimulus category in run 1 were then correlated with multivoxel activity patterns for each stimulus category in run 2 and vice versa. The resulting correlations were then Fisher transformed $\{0.5 \times \ln [(1+r) /(1-r)]\}$ and averaged across the two run-wise comparisons (e.g., hands even - tools odd and tools even - hands odd). The scrambled objects condition was excluded from the analysis because this condition was used as a baseline for ROI definition. The resulting $4 \times 4$ correlation matrix for each subject and ROI provides an estimate of the neural similarity of the four object categories. Correlations were quantified via ANOVAs and pairwise ttests. 


\section{Results}

\section{Single subject ROIs analysis}

Consistent with previous research on the neural representation of tools (Chao and Martin, 2000; Lewis, 2006; Valyear and Culham, 2010; Bracci et al., 2012), the tool contrast (tools > scrambled objects) revealed a network of areas that comprised the IPS, LOTC and VOTC (Figure 2a, purple color-coded) in the left hemisphere. Similarly, the hand contrast (hands > scrambled objects) revealed hand responses - partially overlapping with the tool responses in the left IPS, left LOTC and left VOTC (Figure 2a, blue color-coded). Figure 2a shows partially overlapping responses to hands and tools in the lateral and ventral occipitotemporal and parietal areas in the left hemisphere of four representative participants. Table 1 reports single-subject average peak activation coordinates for these contrasts of interest. It is further noted that we observed handand tool-related activations in the corresponding occipitotemporal areas of the right hemisphere (Supplementary Figure 1a). However, given that overlapping tool- and hand-related activations in the IPS were consistently localized within the left hemisphere only, the following analyses were constrained to the left hemisphere only.

We subsequently examined for possible similarities in the profile of partially overlapping hand and tool regions (Material and Methods), separately for each ROI (VOTC, LOTC and IPS). Parameter estimates for each condition were extracted from each contrast in each individual subject and tested in a $2 \times 4$ ANOVA with Contrast (hand, tool) and Condition (hands, tools, bodies, nonmanipulable objects) as the within-subject factors. Results revealed a significant Contrast $x$ Condition interaction $\left(F_{(3,33)}=8.23 ; p<0.001\right.$, Figure $\left.2 b\right)$ in IPS. Post hoc $t$-tests revealed differential functional profiles for IPS-hand and IPS-tool. In IPS-hand, the hand condition elicited the highest response, as compared to all other categories $(p<0.001$, for all tests). Notably, within the IPS-tool, the response to hands and tools did not differ from each 
other $(p=0.54)$, and both elicited significantly higher response relative to the remaining conditions ( $p<0.03$, for all tests).

In LOTC, the $2 \times 4$ ANOVA with Contrast (hand, tool) and Condition (hands, tools, bodies, nonmanipulable objects) as the within-subject factors revealed a significant Contrast $x$ Condition interaction $\left(F_{(3,42)}=6.13 ; p=0.001\right.$, see Figure $\left.2 b\right)$. Post hoc testing revealed that in both LOTChand and LOTC-tool responses to hands were highest compared to all other categories $(p<0.03$, for all tests). In both ROIs, responses to tools were significantly higher than nonmanipulable objects ( $p<0.002$, for all tests), but were significantly lower than bodies ( $p<0.006$, for all tests).

As for IPS and LOTC, in VOTC the $2 \times 4$ ANOVA with Contrast (hand, tool) and Condition (hands, tools, bodies, nonmanipulable objects) as the within-subject factors revealed a significant interaction of Contrast $\times$ Condition $\left(F_{(3,33)}=21.42 ; p<0.001\right.$, see Figure $\left.2 b\right)$. Post hoc t-tests revealed differential functional profiles for VOTC-hand and VOTC-tool. In VOTC-hand responses to hands and bodies did not differ from each other $(p=0.20)$, and both conditions were significantly higher than the remaining conditions ( $p<0.001$, for all tests). In VOTC-tool responses to all conditions were significantly higher than baseline $(p<0.001$, for all tests), but pairwise comparisons did not reveal differences across all conditions ( $p>0.36$, for all tests). An overview of all post hoc t-tests carried out and their corresponding $p$-values (corrected for the number of comparisons) are listed in Table 2. Single subject ROI analyses for right hemisphere activations are reported in Supplementary Figure 1 and in the Supplementary Results.

Taken together, these results demonstrate that within the left hemisphere of the healthy human cortex, hands and tools activate partially overlapping regions not only in LOTC (Bracci et al., 2012) but also in IPS and VOTC. 


\section{Multivoxel pattern analysis}

Having established the extent of hand/tool overlap by means of the above analysis, we can now further investigate and compare the representational content of the different regions within this network via multivoxel pattern analysis. To frame this in another way: the greater the similarity in the activity patterns of two areas the tighter or more comparable their likely information or computational processing roles. In order to investigate the representational content of parietal and occipitotempomporal hand/tool regions, we compared the distribution of responses to depictions of hands and tools as two object categories that are functionally associated within the action domain, yet largely distinct within the object domain (animate versus inanimate). We predicted high similarity in the distribution of hand and tool response patterns (relative to hands and nonmanipulable objects) in action processing areas. Conversely, in those cortical brain areas that encode category-specific information of objects we would predict response patterns of hands and tools to cluster according to their particular object-domain, respectively (animate and inanimate).

To this aim, we employed correlation-based MVPA (Haxby et al., 2001). As for the ROI analysis, only left hemisphere regions were included in the MVPA analysis. Initial results revealed that for all object categories, within-category (e.g., hands - hands) correlations were always significantly higher than between-category correlations (e.g., hands - bodies; $p<0.001$, for all tests), thus confirming that activity patterns for all categories were reliable and distinguishable from each other within each of the region (Figure 3a). Subsequently, for each ROI (Material and Methods), we quantified the degree to which the representational content is indicative of object category information (animate/inanimate division) and object action information (hand-object action properties). In order to quantify the information with regard to object category, for each ROI, we subtracted the average of the between-domain correlations 
(Figure 3b; light green color-coded cells for correlations between one animate condition and one inanimate condition) from the average of within-domain correlations (Figure 3b; dark green color-coded cells for correlations between either two animate or two inanimate conditions). For each ROI, hand-object action-related information was identified by subtracting the average of hand-object non-action correlations (Figure 3b; light orange color-coded cells for correlations between hands and nonmanipulable objects) from the average of hand-object action-related correlations (Figure 3b; dark orange color-coded cells for correlations between hands and tools). Within-condition correlations (Figure 3b; cells along the diagonal) were excluded from these calculations. The bar graphs presented in Figure 3b depict the degree to which the representational content in left IPS, left LOTC and left VOTC reveals category information (green color-coded) and action information (orange color-coded) of objects.

Subsequently, for each subject and for each ROI, correlations for category information and action information were tested in a further $3 \times 2$ ANOVA with ROI (left IPS, left LOTC, left VOTC) and Information (Category, Action) as within-subject factors. Results revealed a significant ROI $x$ Information interaction $\left(F_{(2,28)}=66.30 ; p<0.001\right.$; Figure $\left.3 \mathrm{~b}\right)$, thus suggesting differences in the information content of each of these ROIs. These conclusions were confirmed with post-hoc analyses, which revealed the following results. In left IPS, action-related information was significantly greater than zero $\left(t_{14}=3.65 ; p=0.003\right)$, whereas information content about object category (animate and inanimate entities) did not significantly differ from zero $\left(t_{14}<1 ; p=0.45\right)$. Furthermore, in this region action-related information was significantly higher than category information $\left(t_{14}=2.13 ; p=0.05\right)$. In contrast, in left LOTC both action and category information was significantly different from zero ( $t_{14}>4 ; p<0.001$, for both tests) and, moreover, these values did not significantly differ from one another $\left(t_{14}<1 ; p=0.50\right)$. Lastly, in left VOTC, the correlation that quantified the categorical information was significantly greater 
compared to the correlation that quantified the action information $\left(t_{14}=14.98 ; p<0.001\right)$, and only information for category was significantly greater than zero $\left(t_{14}=6.24 ; p<0.001\right)$. These results were only observed in the left hemisphere. Refer to Supplementary Figure 2 and the Supplementary Results for the multivoxel pattern analysis for the right hemisphere.

In summary, the present analyses revealed both similarities and differences in the distribution of response patterns to hands and tools across the parietal and occipitotemporal regions in the left hemisphere. Specifically, the activity patterns in IPS cluster according to object action-related information, whereas in VOTC activity patterns cluster most prominently according to category-related information of objects. Most interestingly, LOTC that encodes both the action- and category-related properties of the objects could be argued to act as an effective computational bridge for the parietal and ventral occipitotemporal computations. This organization appears to be specifically left lateralized. 


\section{Discussion}

Functional neuroimaging studies have consistently reported that viewing tools, such as a hammer or a screwdriver, induces activation in the left fronto-parietal and occipitotemporal areas (see for review, Lewis, 2006). The present investigation sought to expand upon these earlier investigations by describing and comparing the representational content of the distinct areas within the tool network. This was accomplished via the identification of any similarities in their response pattern distributions to pictures of tools and hands, compared to other object categories - specifically, bodies and nonmanipulable objects. It is noteworthy that hands and tools differ in visual appearance and object domain, yet these object categories are closely related within the action domain - as both hands and tools are employed in order to carry out skillful and dexterous prehensile actions. Here we predicted that those areas that may be preferentially involved in the processing of hand-object action-related information would show similarities in their subsequent distribution of hand and tool response patterns. Conversely, the distribution of hand and tool representations should dissociate in areas devoted to the processing of category-specific information for animate and inanimate entities.

Initial results from the ROI analysis revealed that, similar to tools, images of hands activated a network of areas in parietal, lateral occipitotemporal and ventral occipitotemporal cortical areas. These results confirm and expand on our previous report (Bracci et al., 2012) showing that hand-related responses were observed not only in left LOTC but also in left IPS and in VOTC.

Furthermore, the results of the MVPA analysis showed that despite hands and tools inducing activation across a similar modular network of parietal and occipitotemporal brain areas, there were some differences in information content in the IPS, LOTC and VOTC. Perhaps most notably, the activity patterns in left LOTC revealed significant information for both action 
and category domains, with high response pattern similarities observed for categories that share action-related properties (hands and tools) but also for entities that correspond to the same object domain (animate and inanimate). Such a functional organization was not observed in the right hemisphere (Supplementary Figure 2). We therefore conclude that left LOTC conjoins these two distinct modular networks (action and objects, specifically) and may represent a computational hub for effective integration across these two functionally distinct cortical processing pathways.

The activity patterns in the left IPS revealed significant information only for object action-related properties. Strikingly, and in contrast to the IPS, VOTC primarily encodes object category-related information. These results provide new insight into the computational role(s) of the regions of the already established tool network. Whereas left IPS is argued to underpin the implementation of tool-hand interactions (Chao and Martin, 2000; Gallivan et al., 2013), VOTC is known to represent aspects of object category-specific knowledge (e.g., animate vs. inanimate; Martin et al., 1996; Martin, 2007; Mahon et al., 2009). Finally, the computational role of left LOTC might be that of associating information processed in VOTC and IPS as here we provide evidence that this cortical area encodes both action- and category-related information, which might be fundamental in order to fully recognize and consequently execute dexterous hand-tool prehensile motor movements. Future work employing functional connectivity analyses could further clarify connectivity patterns among IPS, LOTC and VOTC.

The similarities observed between LOTC and IPS response patterns, were only present in the left hemisphere (Figure 3 and Supplementary Figure 2). Thus, in agreement with clinical studies on tool-use (Johnson-Frey et al., 2005; Buxbaum and Kalenine, 2010; Kalenine et al., 2010; Buxbaum et al., 2014) these results suggest that these areas compute action properties common to both hands and tools. Furthermore, previous literature has provided evidence that 
the left LOTC and the left IPS are computational nodes within a network that underlies skilled hand-object interaction, and when damaged can consequently result in precise deficits in object-specific manipulation skill-sets while nevertheless affording the ability to grasp objects in response to their specific structural content (Sirigu et al., 1995; Buxbaum et al., 2003; letswaart et al., 2006). Further evidence supporting the common involvement of LOTC and IPS in complex hand-object interactions is based on neuroimaging studies. These studies report co-activation of these areas when participants imagine or pantomime tool-use (Grezes and Decety, 2002; Rumiati et al., 2004; Creem-Regehr and Lee, 2005; Johnson-Frey et al., 2005; Arbib et al., 2009), when observing grasping movements toward tools (Creem-Regehr and Lee, 2005), or when planning to grasp either with the hand or with a novel mechanical tool (Arbib et al., 2009). Yet, despite these similarities, representational content in left LOTC and left IPS also revealed differences. The observation that both regions encode object action-related information but only LOTC carries information of object category suggests that the latter might encode -in addition to hand-object action properties - semantic and or/ visual information with relevance for action understanding. Consistent with this idea, neuropsychological evidence has demonstrated that disruption of occipitotemporal areas affects both action-related knowledge in tool-gesture comprehension (i.e. distinguishing between hammering and sawing; Kalenine et al., 2010), and semantic knowledge affecting structural (i.e. body shape) and/or semantic coding of the body (Schwoebel et al., 2004; Moro et al., 2008). Conversely, left parietal lesions can selectively disrupt dynamic coding of body parts' intrinsic positions (Buxbaum et al., 2000) and movement amplitude essential to tool-use (Kalenine et al., 2010).

Tool-use understanding requires computing information with regard to the hand posture and grip configuration relative to a particular tool. This computation requires understanding of whether the hand is correctly shaped in relation to the tool's physical features 
(e.g., handle of a hammer) as well as the tool's specific functional utility. This interpretation is consistent with a recent functional neuroimaging study (Vingerhoets et al., 2013) that reported activation in left LOTC and left anterior IPS when participants were required to judge whether or not a specific hand posture (e.g., precision grip) matched the functional use of a given tool (e.g., car keys). To summarize, the current evidence from the literature together with the present findings suggest a partially common but also a somewhat differential role for left IPS and left LOTC in respect to hand-tool interactions.

Our results are consistent with the vast clinical literature reporting differential impairment of category-specific conceptual knowledge for animate and inanimate objects following focal lesions in ventral temporal cortex (see for review, Caramazza and Shelton, 1998; Capitani et al., 2003). We observed that object responses in VOTC clustered according to object category information. This animate-to-inanimate division that encompasses the ventral surface of the inferior temporal cortex (Konkle and Caramazza, 2013) has been documented across species (Kriegeskorte et al., 2008) and is independent of visual experience (Mahon and Caramazza, 2009). Information content in this region is associated with the specific conceptual knowledge (Martin, 2007), form, size (Haxby et al., 2001; Op de Beeck et al., 2008; Konkle and Oliva, 2012) and surface properties (Cavina-Pratesi et al., 2010b, a) of objects.

Previous imaging studies investigating category selective responses in inferior temporal cortex reported selectivity for faces and bodies in lateral VOTC (Peelen and Downing, 2005; Schwarzlose et al., 2005). Whilst there was a clear significant degree of overlap in cortical activation across these specific categories, high-resolution $\mathrm{fMRI}$ did dissociate functional specificity for these precise categories (Schwarzlose et al., 2005). The present study expands upon these earlier conclusions by demonstrating that selectivity for hands may also be present in VOTC where we report significant within- relative to between-category correlations for both 
hands and bodies (Figure 3a). The observation that the response patterns for these two categories where highly distinguishable in VOTC is in agreement with a recent report that provides support for the idea that both the LOTC and the VOTC house a coherent organization of body part representations (Bracci et al., in press).

As noted in the introduction, selectivity for tools has been consistently reported both in ventromedial OTC (Martin et al., 1996; Chao et al., 1999; Mahon et al., 2007) and in lateral OTC (Chao et al., 1999; Beauchamp et al., 2002, 2003; Bracci et al., 2012; Bracci and Peelen, 2013). The present findings demonstrate differences in the representational/computational content/roles of these regions. While VOTC processes information with regard to the object category, LOTC processes information for both action- and category-related properties of objects. One possible argument for the latter finding is that in LOTC the representations of objects, we perform actions with, partially overlap with the hand representations because of their close relationship with one another in both the action domain (both hands and tools participate in goal directed actions) and the object domain (tools can become an extension of the body; Bracci and Peelen 2013).

In conclusion, we report a comprehensive network of overlapping hand-related and tool-related responses comprising of LOTC, IPS and VOTC in the left hemisphere. Interestingly, MVPA revealed a distinct pattern of representational content for each of these regions, thus suggesting differential computational roles for regions of this network. Specifically, while parietal regions are involved in the processing of hand-object action-related information, ventral occipitotemporal cortical areas are involved in the processing of category-specific information. Remarkably, computations in LOTC by encoding both types of object information may represent a computational hub for effective integration across ventro occipitotemporal perception-related and parietal action-related representations. 


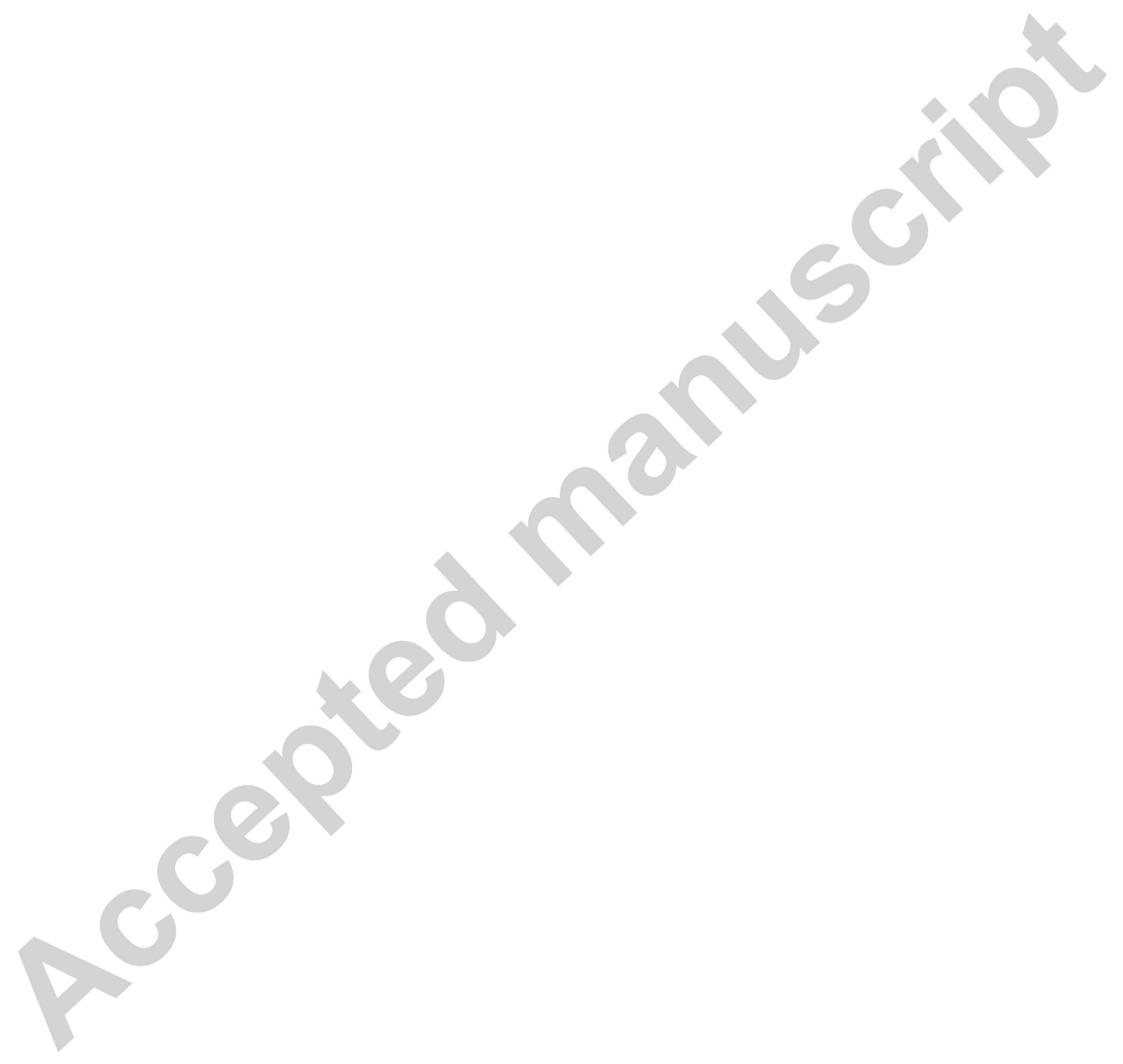


Table 1. Single-subject mean Talairach coordinates for the hand and the tool contrast. Single-subject mean Talairach coordinates (see Figure 2a) are reported for the hand contrast [(hands $>$ scrambled objects) and the tool contrast [(tools $>$ scrambled objects) ( $p=0.005$ uncorrected).

\begin{tabular}{lccc}
\hline Contrasts & $\mathrm{x}$ & $\mathrm{y}$ & $\mathrm{z}$ \\
\hline Hands > scrambled objects & & & \\
Left IPS & -36 & -45 & 46 \\
Left LOTC & -46 & -72 & -5 \\
Left VOTC & -42 & -46 & -17 \\
Tools > scrambled objects & & & \\
Left IPS & -41 & -42 & 43 \\
Left LOTC & -46 & -74 & -8 \\
Left VOTC & -38 & -47 & -19 \\
& & & \\
\hline
\end{tabular}


Table 2: Individual ROI analysis statistical overview. Overview of individual ROI parameter estimates pairwise t-tests including $\mathrm{t}$-values and uncorrected $p$-values for each ROI (IPS-hand, IPS-tool, LOTC-hand, LOTC-tool, VOTC-hand, VOTC-tool). Bold characters indicate $p$-values that survived correction for multiple comparisons. Only contrasts for hands and tools relative to the remaining conditions (nonmanipulable objects and bodies) are reported.

ROIs

Conditions

\begin{tabular}{|c|c|c|c|c|}
\hline & & Tools & Bodies & nmanipulable objects \\
\hline \multirow[t]{2}{*}{ IPS-hand } & Hands & $\mathrm{t}=8.90 ; p<\mathbf{0 . 0 0 1}$ & $\mathrm{t}=9.52 ; p<0.001$ & $\mathrm{t}=14.92 ; p<0.001$ \\
\hline & Tools & & $\mathrm{t}=7.50 ; p<0.001$ & $\mathrm{t}=2.27 ; p=0.045$ \\
\hline \multirow[t]{2}{*}{ IPS-tool } & Hands & $\mathrm{t}=0.63 ; p=0.540$ & $\mathrm{t}=2.53 ; p=0.028$ & $\mathrm{t}=2.89 ; p=0.015$ \\
\hline & Tools & & $\mathrm{t}=3.82 ; p=0.003$ & $\mathrm{t}=4.67 ; p=\mathbf{0 . 0 0 1}$ \\
\hline \multirow[t]{2}{*}{ LOTC-hand } & Hands & $\mathrm{t}=12.09 ; p<0.001$ & $\mathrm{t}=3.55 ; p=\mathbf{0 . 0 0 3}$ & $\mathrm{t}=11.95 ; p<\mathbf{0 . 0 0 1}$ \\
\hline & Tools & & $\mathrm{t}=-6.01 ; p<0.001$ & $\mathrm{t}=4.34 ; p=\mathbf{0 . 0 0 1}$ \\
\hline \multirow[t]{2}{*}{ LOTC-tool } & Hands & $\mathrm{t}=7.37 ; p<0.001$ & $\mathrm{t}=2.31 ; p=0.036$ & $\mathrm{t}=9.24 ; p<0.001$ \\
\hline & Tools & & $\mathrm{t}=-3.21 ; p=0.006$ & $\mathrm{t}=3.75 ; p=\mathbf{0 . 0 0 2}$ \\
\hline \multirow[t]{2}{*}{ VOTC-hand } & Hands & $\mathrm{t}=10.37 ; p<0.001$ & $\mathrm{t}=1.36 ; p=0.198$ & $\mathrm{t}=5.93 ; p<\mathbf{0 . 0 0 1}$ \\
\hline & Tools & & $\mathrm{t}=-6.49 ; p<\mathbf{0 . 0 0 1}$ & $\mathrm{t}=0.72 ; p=0.483$ \\
\hline \multirow[t]{2}{*}{ VOTC-tool } & Hands & $\mathrm{t}=0.66 ; p=0.522$ & $\mathrm{t}=-0.42 ; p=0.688$ & $\mathrm{t}=-0.29 ; p=0.773$ \\
\hline & Tools & & $\mathrm{t}=-0.95 ; p=0.365$ & $\mathrm{t}=-0.55 ; p=0.596$ \\
\hline
\end{tabular}




\section{Figure captions}

Figure 1. Experimental stimuli. For each experimental condition (nonmanipulable objects, tools, hands, bodies, and scrambled objects) 6 (out of 70) images are presented.

Figure 2. Individual-subject activation maps and ROI analysis for the hand and tool contrast. A) Individual-subject hand-responsive regions (blue color-coded; [hands > scrambled objects]), and tool-responsive regions (purple color-coded; [tools > scrambled objects]) are shown in the left hemisphere of four representative participants at the threshold $p=0.005$ uncorrected. B) Mean estimates for nonmanipulable objects, tools, hands and bodies are shown in left IPS (hand and tool), left LOTC (hand and tool) and left VOTC (hand and tool). ROIs were restricted to a cube of $20 \mathrm{~mm}$ width centered on the activation peak (threshold $p<0.005$, uncorrected). Error bars indicate SEM.

Figure 3. Multivoxel pattern analysis (MVPA) in the left hemisphere. A) Multivoxel correlation matrices in left IPS, left LOTC and left VOTC, as functionally defined in each individual subject upon contrasting the average response to all object categories (nonmanipulable objects, tools, hands and bodies) relative to scrambled objects. Each cell of the matrix represents a correlation value (averaged across subjects). B) Bar graphs show category information and action information in IPS, LOTC and VOTC in the left hemisphere. Information about object category was computed by subtracting the average of between-object domain correlations (light green color-coded cells for correlations between one animate condition and one inanimate condition) from the average of within-object domain correlations (dark green color-coded cells for correlations between either two animate or two inanimate conditions). Information about object action was computed by subtracting the average of hand-object non-action correlations (light orange color-coded cells for correlations between hands and nonmanipulable objects) from the average of hand-object action-related correlations (dark orange color-coded cells for correlations between hands and tools). Error bars indicate SEM. 


\section{Supplementary Figure 1. Individual-subject activation maps and ROI analysis for the} hand and tool contrast in the right hemisphere. A) Individual-subject hand-responsive regions (blue color-coded; [hands > scrambled objects]), and tool-responsive regions (purple color-coded; [tools > scrambled objects]) are shown in the right hemisphere of four representative participants at the threshold $p=0.005$ uncorrected. B) Mean estimates for nonmanipulable objects, tools, hands and bodies are shown in left LOTC (hand and tool) and left VOTC (hand and tool). ROls were restricted to a cube of $20 \mathrm{~mm}$ width centered on the activation peak (threshold $p<0.005$, uncorrected). Error bars indicate SEM.

\section{Supplementary Figure 2. Multivoxel pattern analysis (MVPA) in the right hemisphere.}

A) Multivoxel correlation matrices in right IPS, right LOTC and right VOTC, as functionally defined in each individual subject upon contrasting the average response to all object categories (nonmanipulable objects, tools, hands and bodies) relative to scrambled objects. Each cell of the matrix represents a correlation value (averaged across subjects). B) Bar graphs show category information and action information in IPS, LOTC and VOTC in the right hemisphere. Information about object category was computed by subtracting the average of between-object domain correlations (light green color-coded cells for correlations between one animate condition and one inanimate condition) from the average of within-object domain correlations (dark green color-coded cells for correlations between either two animate or two inanimate conditions). Information about object action was computed by subtracting the average of hand-object non-action correlations (light orange color-coded cells for correlations between hands and nonmanipulable objects) from the average of hand-object action-related correlations (dark orange color-coded cells for correlations between hands and tools). Error bars indicate SEM. 


\section{References}

Almeida J, Fintzi AR, Mahon BZ (2013) Tool manipulation knowledge is retrieved by way of the ventral visual object processing pathway. Cortex 49:2334-2344.

Andersen RA, Kellis S, Klaes C, Aflalo T (2014) Toward more versatile and intuitive cortical brain-machine interfaces. Current Biology 24:R885-897.

Arbib MA, Bonaiuto JB, Jacobs S, Frey SH (2009) Tool use and the distalization of the end-effector. Psychol Res 73:441-462.

Beauchamp MS, Lee KE, Haxby JV, Martin A (2002) Parallel visual motion processing streams for manipulable objects and human movements. Neuron 34:149-159.

Beauchamp MS, Lee KE, Haxby JV, Martin A (2003) FMRI responses to video and pointlight displays of moving humans and manipulable objects. J Cogn Neurosci 15:991-1001. Beauchamp MS, Lee KE, Argall BD, Martin A (2004) Integration of auditory and visual information about objects in superior temporal sulcus. Neuron 41:809-823.

Bracci S, Peelen MV (2013) Body and object effectors: the organization of object representations in high-level visual cortex reflects body-object interactions. The Journal of Neuroscience 33:18247-18258.

Bracci S, Cavina-Pratesi C, letswaart M, Caramazza A, Peelen MV (2012) Closely overlapping responses to tools and hands in left lateral occipitotemporal cortex. Journal of Neurophysiology 107:1443-1456.

Brainard DH (1997) The Psychophysics Toolbox. Spat Vis 10:433-436.

Buxbaum L, Kalenine S (2010) Action knowledge, visuomotor activation, and embodiment in the two action systems. Ann N Y Acad Sci 1191:201-218.

Buxbaum L, Giovannetti T, Libon D (2000) The role of the dynamic body schema in praxis: evidence from primary progressive apraxia. Brain Cogn 44:166-191.

Buxbaum L, Shapiro AD, Coslett HB (2014) Critical brain regions for tool-related and imitative actions: a componential analysis. Brain 137:1971-1985.

Buxbaum L, Sirigu A, Schwartz MF, Klatzky R (2003) Cognitive representations of hand posture in ideomotor apraxia. Neuropsychologia 41:1091-1113.

Buxbaum L, Kyle K, Grossman M, Coslett HB (2007) Left inferior parietal representations for skilled hand-object interactions: evidence from stroke and corticobasal degeneration. Cortex 43:411-423.

Capitani E, Laiacona M, Mahon B, Caramazza A (2003) What are the facts of semantic category-specific deficits? A critical review of the clinical evidence. Cognitive Neuropsychology 20:213-261.

Caramazza A, Shelton JR (1998) Domain-specific knowledge systems in the brain the animate-inanimate distinction. Journal of Cognitive Neuroscience 10:1-34.

Cavina-Pratesi C, Kentridge RW, Heywood CA, Milner AD (2010a) Separate channels for processing form, texture, and color: evidence from FMRI adaptation and visual object agnosia. Cereb Cortex 20:2319-2332.

Cavina-Pratesi C, Kentridge RW, Heywood CA, Milner AD (2010b) Separate processing of texture and form in the ventral stream: evidence from FMRI and visual agnosia. Cereb Cortex 20:433-446. 
Chao LL, Martin A (2000) Representation of manipulable man-made objects in the dorsal stream. Neuroimage 12:478-484.

Chao LL, Haxby JV, Martin A (1999) Attribute-based neural substrates in temporal cortex for perceiving and knowing about objects. Nature neuroscience 2:913-919.

Choi SH, Na DL, Kang E, Lee KM, Lee SW, Na DG (2001) Functional magnetic resonance imaging during pantomiming tool-use gestures. Experimental Brain Research 139:311317.

Creem-Regehr SH, Lee JN (2005) Neural representations of graspable objects: are tools special? Brain Res 22:457-469.

Creem-Regehr SH, Willemsen P, Gooch AA, Thompson WB (2005) The influence of restricted viewing conditions on egocentric distance perception: implications for real and virtual indoor environments. Perception 34:191-204.

Damasio H, Tranel D, Grabowski T, Adolphs R, Damasio A (2004) Neural systems behind word and concept retrieval. Cognition 92:179-229.

Downing PE, Chan AW, Peelen MV, Dodds CM, Kanwisher N (2006) Domain specificity in visual cortex. Cereb Cortex 16:1453-1461.

Gallivan JP, McLean DA, Valyear KF, Culham JC (2013) Decoding the neural mechanisms of human tool use. Elife 2:e00425.

Grezes J, Decety J (2002) Does visual perception of object afford action? Evidence from a neuroimaging study. Neuropsychologia 40:212-222.

Haxby JV, Gobbini MI, Furey ML, Ishai A, Schouten JL, Pietrini P (2001) Distributed and overlapping representations of faces and objects in ventral temporal cortex. Science 293:2425-2430.

letswaart M, Carey DP, Della Sala S (2006) Tapping, grasping and aiming in ideomotor apraxia. Neuropsychologia 44:1175-1184.

Johnson-Frey SH, Newman-Norlund R, Grafton ST (2005) A distributed left hemisphere network active during planning of everyday tool use skills. Cerebral Cortex 15:681-695.

Kalenine S, Buxbaum $\amalg$, Coslett HB (2010) Critical brain regions for action recognition: lesion symptom mapping in left hemisphere stroke. Brain 133:3269-3280.

Kellenbach ML, Brett M, Patterson K (2003) Actions speak louder than functions: the importance of manipulability and action in tool representation. Journal of Cognitive Neuroscience 15:30-46.

Konkle T, Oliva A (2012) A real-world size organization of object responses in occipitotemporal cortex. Neuron 74:1114-1124.

Konkle T, Caramazza A (2013) Tripartite organization of the ventral stream by animacy and object size. The Journal of Neuroscience 33:10235-10242.

Kriegeskorte N, Mur M, Ruff DA, Kiani R, Bodurka J, Esteky H, Tanaka K, Bandettini PA (2008) Matching categorical object representations in inferior temporal cortex of man and monkey. Neuron 60:1126-1141.

Lewis JW (2006) Cortical networks related to human use of tools. Neuroscientist 12:211231.

Mahon BZ (2013) Watching the brain in action. Elife 2:e00866.

Mahon BZ, Caramazza A (2009) Concepts and categories: a cognitive neuropsychological perspective. Annu Rev Psychol 60:27-51. 
Mahon BZ, Kumar N, Almeida J (2013) Spatial frequency tuning reveals interactions between the dorsal and ventral visual systems. Journal of Cognitive Neuroscience 25:862-871.

Mahon BZ, Anzellotti S, Schwarzbach J, Zampini M, Caramazza A (2009) Categoryspecific organization in the human brain does not require visual experience. Neuron 63:397-405.

Mahon BZ, Milleville SC, Negri GA, Rumiati RI, Caramazza A, Martin A (2007) Actionrelated properties shape object representations in the ventral stream. Neuron 55:507520.

Martin A (2007) The representation of object concepts in the brain. Annual Review of Psychology 58:25-45.

Martin A, Wiggs CL, Ungerleider LG, Haxby JV (1996) Neural correlates of categoryspecific knowledge. Nature 379:649-652.

Moro V, Urgesi C, Pernigo S, Lanteri P, Pazzaglia M, Aglioti SM (2008) The neural basis of body form and body action agnosia. Neuron 60:235-246.

Oldfield RC (1971) The assessment and analysis of handedness: the Edinburgh inventory. Neuropsychologia 9:97-113.

Op de Beeck HP, Torfs K, Wagemans J (2008) Perceived shape similarity among unfamiliar objects and the organization of the human object vision pathway. J Neurosci 28:10111-10123.

Orlov T, Makin T, Zohary E (2010) Topographic representation of the human body in the occipitotemporal cortex. Neuron 4:586-600.

Peelen MV, Downing PE (2005) Selectivity for the human body in the fusiform gyrus. J Neurophysiol 93:603-608.

Peelen MV, Bracci S, Lu X, He C, Caramazza A, Bi Y (2013) Tool Selectivity in Left Occipitotemporal Cortex Develops without Vision. Journal of Cognitive Neuroscience 25:1225-1234.

Rumiati RI, Weiss PH, Shallice T, Ottoboni G, Noth J, Zilles K, Fink GR (2004) Neural basis of pantomiming the use of visually presented objects. Neuroimage 21:1224-1231.

Schwarzlose RF, Baker Cl, Kanwisher N (2005) Separate face and body selectivity on the fusiform gyrus. J Neurosci 25:11055-11059.

Schwoebel J, Buxbaum LJ, Coslett HB (2004) Representations of the human body in the production and imitation of complex movements. Cognitive neuropsychology 21:285298.

Sirigu A, Duhamel JR, Poncet M (1991) The role of sensorimotor experience in object recognition. A case of multimodal agnosia. Brain 114 ( Pt 6):2555-2573.

Sirigu A, Cohen L, Duhamel JR, Pillon B, Dubois B, Agid Y (1995) A selective impairment of hand posture for object utilization in apraxia. Cortex; a journal devoted to the study of the nervous system and behavior 31:41-55.

Tranel D, Kemmerer D, Adolphs R, Damasio H, Damasio AR (2003) Neural correlates of conceptual knowledge for actions. Cognitive Neuropsychology:409-432.

Valyear KF, Culham JC (2010) Observing learned object-specific functional grasps preferentially activates the ventral stream. J Cogn Neurosci 22:970-984. 
Vingerhoets G, Nys J, Honore P, Vandekerckhove E, Vandemaele P (2013) Human left ventral premotor cortex mediates matching of hand posture to object use. PLoS One 8:e70480.

1) MVPA showed representational content differences in regions within the tool network.

2) Left parietal regions encode hand-tool action-related information.

3) Ventral OTC of the tool-network encodes object category information.

4) Left lateral OTC encodes hand-tool action-related and category-related information.

5) Left lateral OTC bridges ventral OTC (category) and parietal (action) representations. 

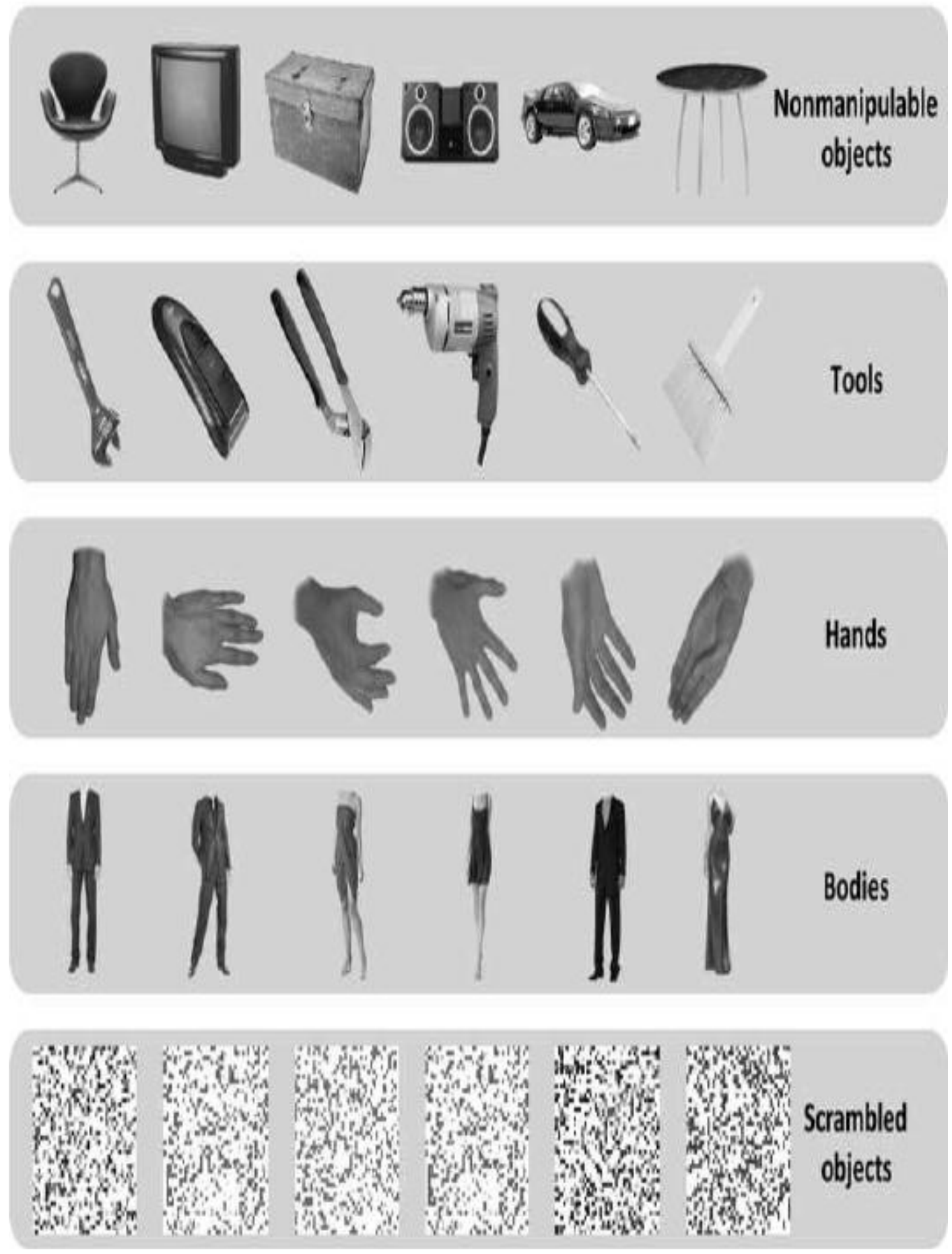
A
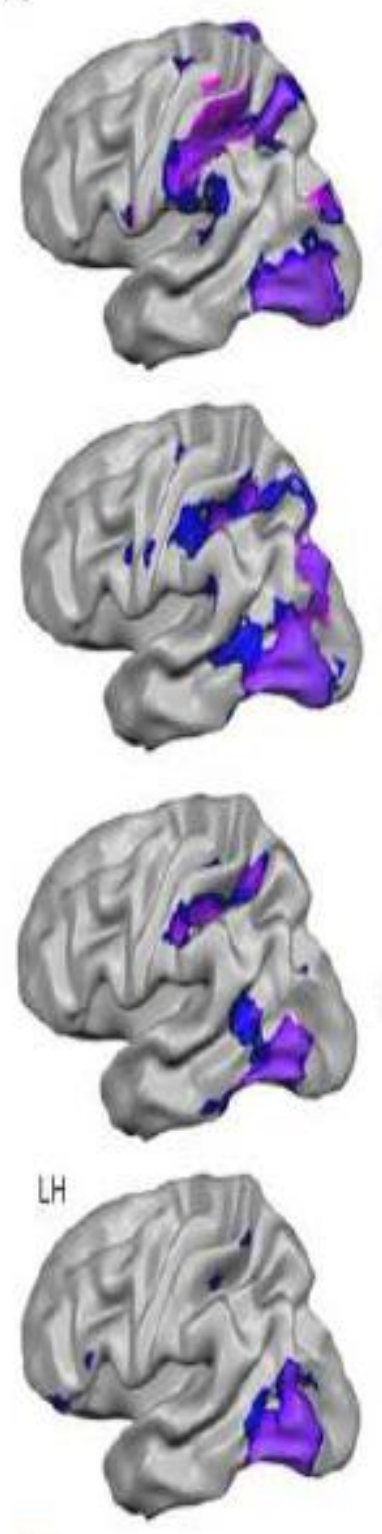

hands > scrambled objects
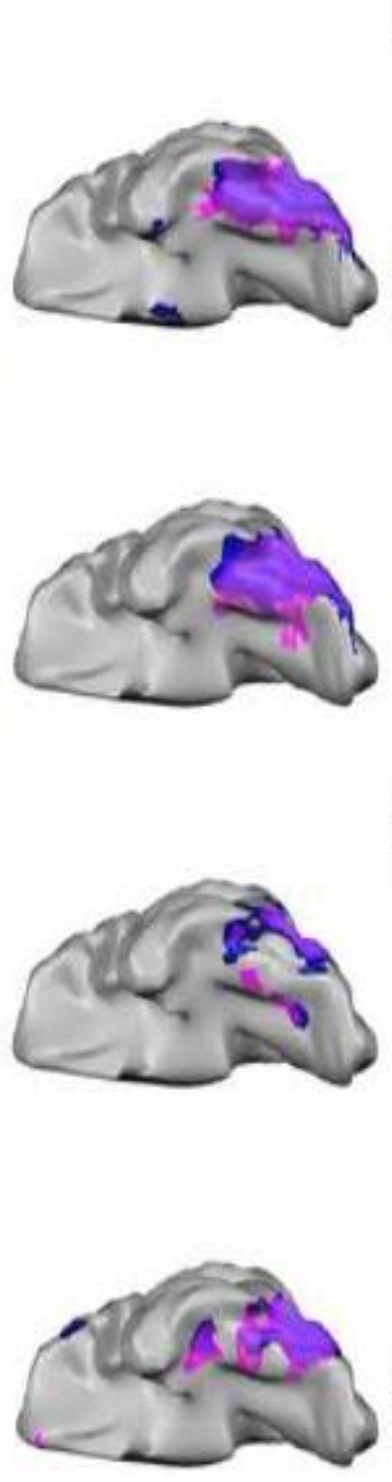

tools > scrambled objects
B
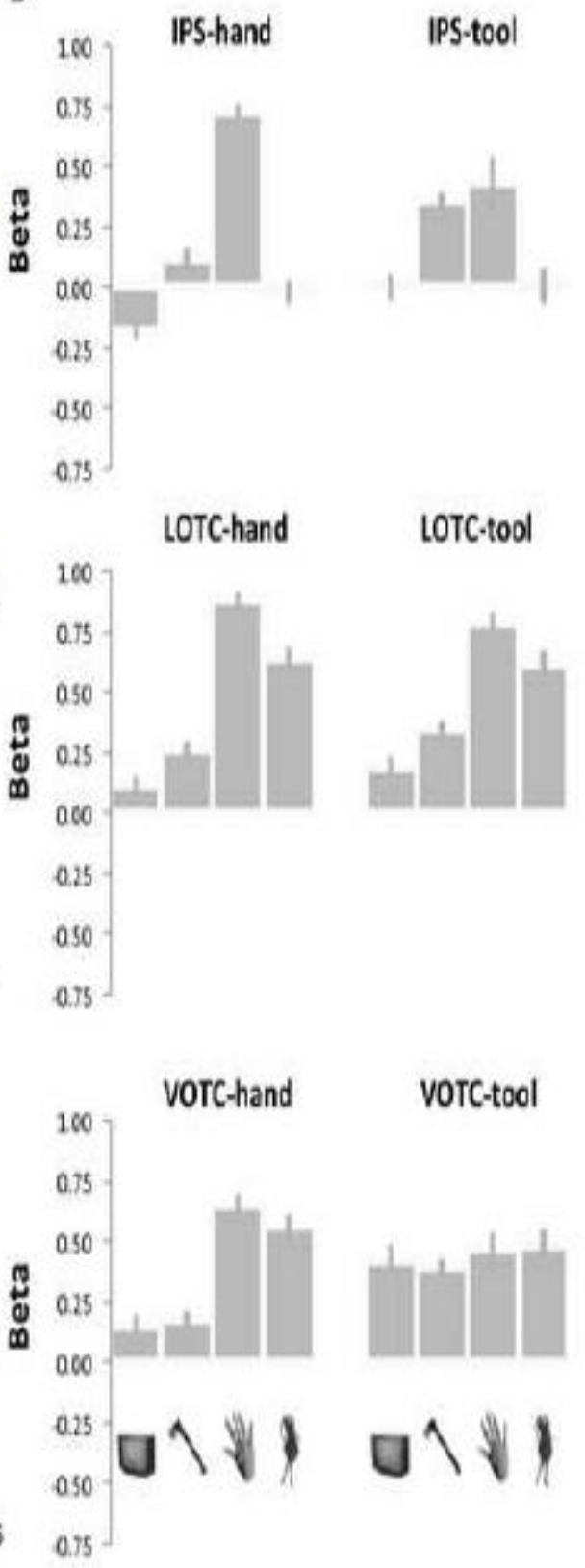


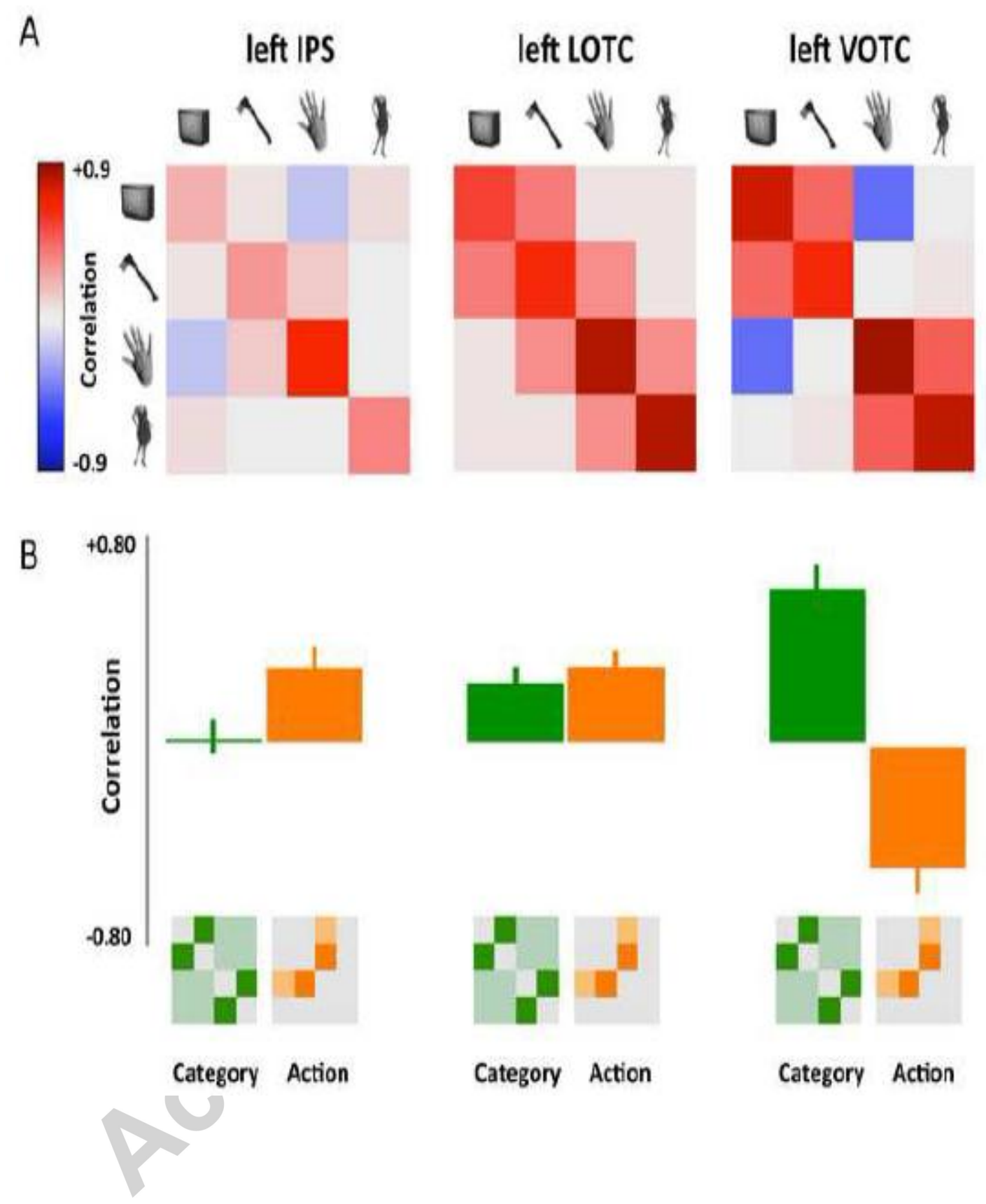

